\title{
The formation of a sunspot penumbra ${ }^{\star}$
}

\author{
R. Schlichenmaier, R. Rezaei, N. Bello González, and T. A. Waldmann
}

Kiepenheuer-Institut für Sonnenphysik, Schöneckstr. 6, 79104 Freiburg, Germany

e-mail: [schliche;rrezaei;nbello; waldmann]@kis.uni-freiburg.de

Received 21 January 2010 / Accepted 18 February 2010

\begin{abstract}
Context. The formation of a penumbra is crucial for our understanding of solar magnetism, but it has not been observed in detail. Aims. We aim to enhance our knowledge of how a sunspot penumbra forms and how sunspots grow in size.

Methods. We present a data set of the active region NOAA 11024 acquired at the German VTT with speckle-reconstructed images in the G-band and Ca II K. The data set includes spectropolarimetric profiles from GFPI in Fe I 617.3 nm and TIP in Fe I $1089.6 \mathrm{~nm}$.

Results. On 2009 July 4, at 08:30 UT, a leading spot without penumbra and pores of opposite polarity were present in the active region. For the next 4:40 h, we observed the formation of a penumbra in the leading spot at a cadence of 5 images per second. We produced speckle reconstructed images of 0.'3 spatial resolution or better, interrupted by one large gap of 35 min and a few more small gaps of about $10 \mathrm{~min}$. The leading spot initially has a size of $230 \operatorname{arcsec}^{2}$ with only a few penumbral filaments and then grows to a size of $360 \operatorname{arcsec}^{2}$. The penumbra forms in segments, and it takes about $4 \mathrm{~h}$ until it encircles half of the umbra, on the side opposite the following polarity. On the side towards the following polarity, elongated granules mark a region of magnetic flux emergence.

Conclusions. This ongoing emergence appears to prevent a steady penumbra from forming on this side. While the penumbra forms, the umbral area is constant; i.e., the increase in the total spot area is caused exclusively by the growth of the penumbra. From this we conclude that the umbra has reached an upper size limit and that any new magnetic flux that joins the spot is linked to the process of penumbral formation.
\end{abstract}

Key words. sunspots - Sun: activity - magnetic fields - Sun: photosphere

\section{Introduction}

The generation of magnetic fields in the solar interior and the emergence processes of magnetic flux through the convection zone into the photosphere and on into the outer atmosphere severely challenge our understanding of solar magnetohydrodynamics. Sunspots are the most prominent example of solar magnetic fields in the photosphere. The event of flux emergence together with the formation of sunspots represents a crucial episode of the full problem.

Solanki (2003) reviews our understanding of sunspot formation and relies on reviews by Zwaan $(1987,1992)$. Intriguing data sets on the formation of penumbrae have been presented by Leka \& Skumanich (1998) and Yang et al. (2003). Both papers find (i) that the formation of penumbral filaments takes place on a time scale of about a half an hour; (ii) that it is associated with more inclined magnetic fields in penumbral areas; and (iii) that the Evershed flow is intimately coupled with inclined magnetic fields. Leka \& Skumanich observe that the penumbra forms in segments and gradually grows around the proto-spot. However, those pioneering data have low spatial and temporal resolution. Yang et al. report on images with better spatial resolution, but observe only a penumbral segment that forms as an extension of a light bridge and which does not grow to circle the spot.

We have witnessed the formation of penumbra with a high temporal cadence for $4.5 \mathrm{~h}$ at high spatial resolution. Here we present this unique data set, and put our observation in the context of current knowledge.

^ Movies are only available in electronic form at http: //www . aanda. org

\section{Observations}

The evolution of the active region NOAA 11024 was observed on consecutive days from July 1 until July 10, 2009. The most interesting part of our data set is a $4: 40 \mathrm{~h}$ sequence when a sunspot developed a penumbra. The observations were taken at the German Vacuum Tower Telescope (VTT) with a multiwavelength set-up: (1) image bursts with filters in G-band (1 nm bandwidth at $403 \mathrm{~nm}$, exposure time of $7.5 \mathrm{~ms})$ and Ca II K (1 nm at $393 \mathrm{~nm}, 20 \mathrm{~ms}$ ) with a spatial sampling of $0 .^{\prime} 07$; (2) spectropolarimetric GFPI data (Puschmann et al. 2006; Bello González \& Kneer 2008) with 31 wavelength steps in Fe I $617.3 \mathrm{~nm}$; and (3) scans with the spectro-polarimeter TIP II (Collados et al. 2007; Schlichenmaier \& Collados 2002) in Fe I 1089.6 nm. The observations benefited from the adaptive optics system KAOS (von der Lühe et al. 2003).

In this paper, we concentrate on the imaging data (G-band and Ca II K) that was reconstructed using the speckleinterferometry package KISIP (Wöger \& von der Lühe 2008), in "triple correlation" mode. We recorded 5 frames per second and selected 100 frames every 35 s to produce a speckle reconstructed image, which has a spatial resolution of better than 0.3 for the G-band ${ }^{1}$. Due to periods of bad seeing, our time series has one interruption of 35 min between 11:12 and 11:47 UT and a few more interruptions each shorter than $10 \mathrm{~min}$. The data gaps are seen in Fig. 4 as missing data points for the values of umbral area.

\footnotetext{
1 Animations of the speckle-reconstructed Ca II K and G-band filtergram sequences can be downloaded as online material to this letter.
} 

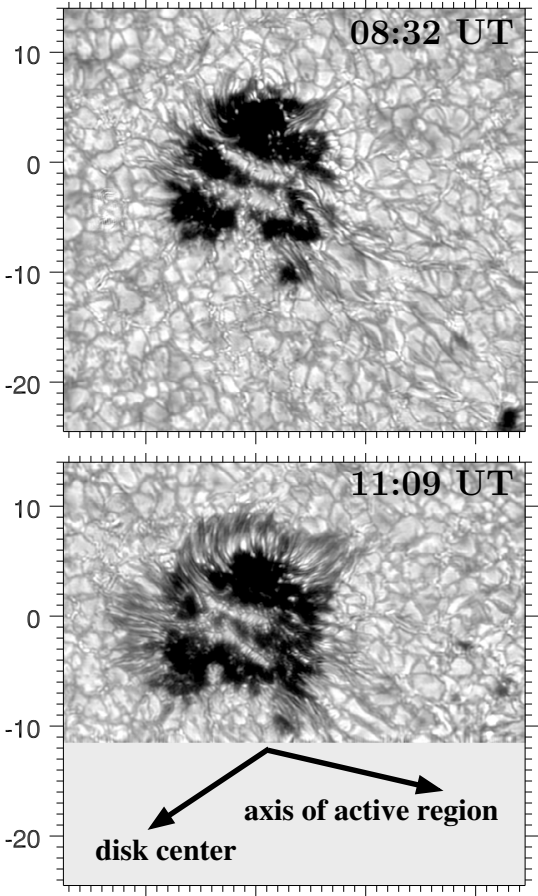

$-10$ 0
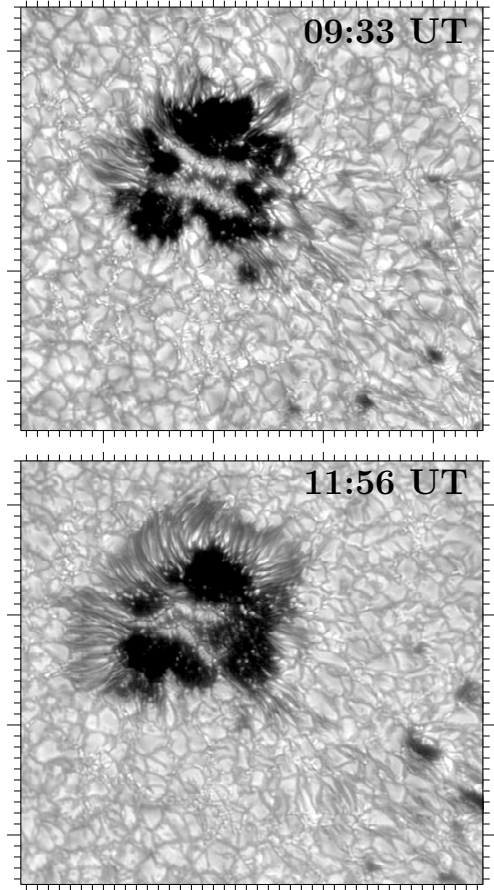

$-10$

0

10

20
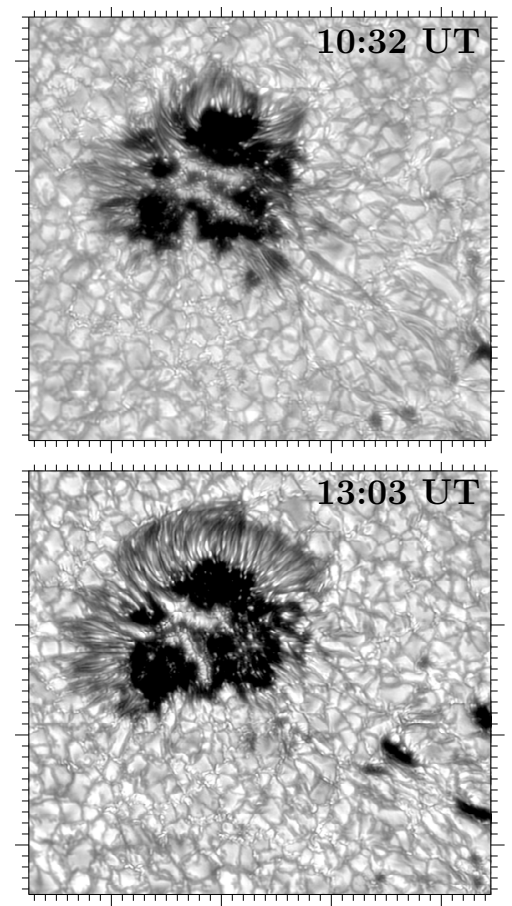

$\begin{array}{lll}-10 & 0 & 10\end{array}$

20

Fig. 1. Snapshots of the spot on 2009 July 4 as it is developing a penumbra. The spot is located at $25^{\circ} \mathrm{S}$ and migrates with solar rotation from $7^{\circ} \mathrm{E}$ at 08:32 UT to $5^{\circ} \mathrm{E}$ at 13:03 UT. The three little pores at the lower right of the image at 11:56 UT have the same polarity as the spot. Tick marks correspond to $1^{\prime \prime}$ and the axes are relative to spot center. In the lower left panel, one arrow points towards disk center and the other points along the direction towards the center of the opposite polarity, as seen from spot center.
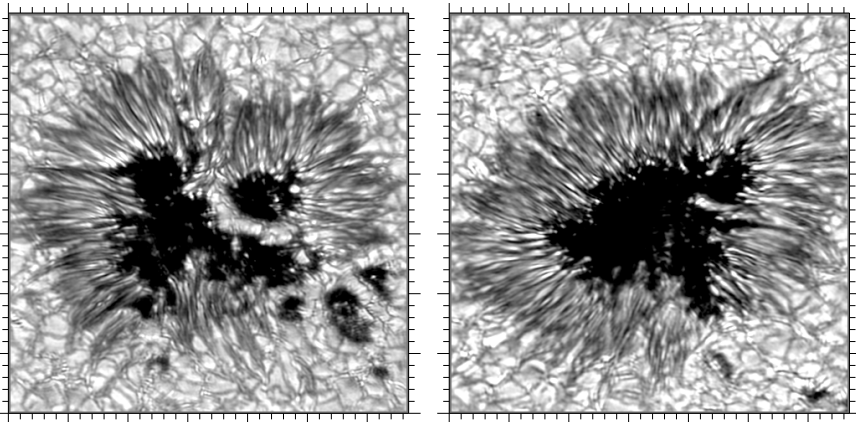

Fig. 2. Snapshots of evolving sunspot on July 5th (left panel) at $8^{\circ} \mathrm{W} /$ $24^{\circ} \mathrm{S}$ and July 6 th (right panel) at $23^{\circ} \mathrm{W} / 24^{\circ} \mathrm{S}$. Tick marks correspond to $1^{\prime \prime}$.

\section{Results}

\subsection{Global evolution of NOAA 11024}

We started to observe NOAA 11024 on July 1 . It appeared as a facular region developing two pores at $52^{\circ} \mathrm{E} / 25^{\circ} \mathrm{S}$. From TIP maps, we measured that these pores have opposite polarities. They had a size of a little more than $2^{\prime \prime}$ and were separated by $10^{\prime \prime}$. They dissolved until July 2 , and the active region again showed only faculae. In the morning of July 4 at 08:30 UT, a spot and pores of opposite polarity separated by about $30^{\prime \prime}$ were observed. The sunspot we observed had a diameter of about $17^{\prime \prime}$. We call it a sunspot because of its large size, even though it does not exhibit a fully developed penumbra (cf., Keppens \& Martínez Pillet 1996). In addition, we did not observe a clear inward motion of granules, a typical signature of pores (Sobotka 2003). It was located at $7^{\circ} \mathrm{E} / 25^{\circ} \mathrm{S}$, and was leading the
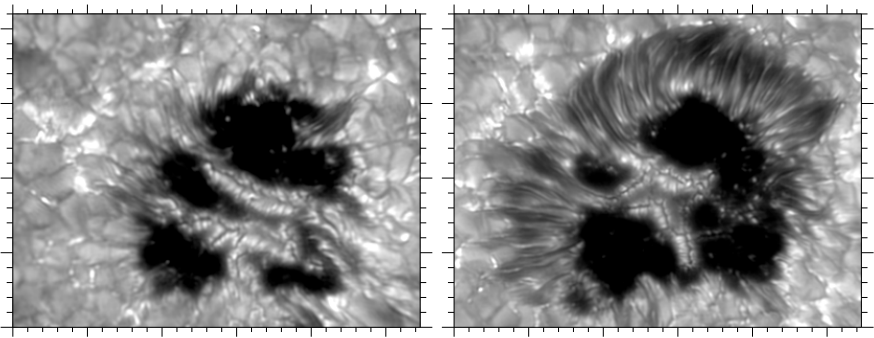

Fig. 3. Imaging the spot in the Ca II K line at $393 \mathrm{~nm}$ revealing dark grooves along the middle of the light bridges. These dark cores are seen more clearly than in the G-band images. The left and right panels show two snapshots at 08:33 UT and 12:42 UT, respectively. Tick marks correspond to $1^{\prime \prime}$.

active region with respect to the direction of rotation. It developed a penumbra between 8:30 UT and 13:10 UT (cf., Fig. 1 and Sect. 3.2). This forming sunspot evolved into a mature sunspot ${ }^{2}$. Figure 2 illustrates that the penumbra almost encircles the spot on July 5, and that the light bridges disappeared until July 6 . The light bridges partially reappeared on later days. The sunspot could be observed until it reached the western limb on July 11. The magnetic flux of the opposite (following) polarity of this active region also merged, but it took until July 8 for a second sunspot to form, reflecting a typical behavior of active regions (e.g., Strous et al. 1996; Martínez Pillet 2002). At that time the two centers of opposite polarity were separated by more than $100^{\prime \prime}$. On July 4, the active region had a tilt angle of some $25^{\circ}$

2 The further evolution of the active region starting at about 12:00 UT on July 4 was also observed with the SOT telescope onboard Hinode, as well as with MDI on SOHO. 
with the leading polarity closer to the equator, in accordance with Joy's law (Hale et al. 1919). It is also interesting to note that the remnants of the active region reappeared on the eastern limb on July 26, as seen in MDI images.

\subsection{The sunspot development on July 4}

Our observing sequence starts at 08:30 UT, when the spot is characterized by (a) two prominent light bridges, and (b) a few peripheral filaments that later evolve into penumbral filaments and subsequently into penumbral segments. In the lower left panel of Fig. 1, an arrow indicates the direction towards the following polarity. In that direction, the granulation is characterized by elongated filamentary features, i.e., elongated granules (cf. Bray \& Loughhead 1964). The elongated granules are oriented along the axis of the active region.

\subsubsection{Morphology and evolution of light bridges}

The snapshots in Figs. 1-3 monitor the change of the shape of the light bridges during our observations. The two light bridges that are separated at 08:32 UT are connected by an additional light bridge at 09:33 UT and continue to evolve.

The light bridges display dark grooves along their central lanes, which are more pronounced in Ca II (cf., Fig. 3) than in the G-band. These dark cores are flanked by small elongated granule-like cells with a size of approximately $1^{\prime \prime}$ by 0.3 . The last line up almost perpendicular to the dark core of the light bridge, and are separated from each other by 0.5, fragmenting and merging within 1 to $3 \mathrm{~min}$. In the Ca II filtergrams, the light bridge exhibits branches, with the dark central lanes connected. The light bridges change their appearance in the outer umbra; there they either disappear and are replaced by an ensemble of umbral dots or form elongated penumbral filaments. The light bridges are roughly aligned with the axis of the active region. This tendency seems to be typical if one looks at active region image archives, e.g., the DOT archive at http://dotdb.phys.uu.nl/DOT/ (Rutten et al. 2004).

\subsubsection{Formation of a penumbra}

Figure 1 shows consecutive snapshots of the penumbral formation phase. The upper left image shows the spot at 08:32 UT. In this state it exhibits two prominent light bridges and a few distinct (penumbral) filaments at its outer boundary. The most prominent areas of penumbral filamentation are (i) at the lower right of the spot, at about 5 o'clock (with the spot being a clock face, $\left.(x, y) \approx\left(4^{\prime \prime},-8^{\prime \prime}\right)\right)$; and (ii) at about 9:30, 10:30, 11:30, and 2:00 o' clock. In case (i) we can distinguish three filaments. These filaments point towards the opposite polarity of the active region. During the following phase of flux emergence, these filaments do not become larger or more numerous in this section. The filaments of case (ii) do increase their radial extension and become more numerous such that they span a wider and wider azimuthal range as the evolution proceeds.

Except for one, the filaments of case (ii) have a similar shape to filaments of a fully developed penumbra that intrude into the umbra. The one exception is at 11:30 o'clock $\left(-2^{\prime \prime}, 7^{\prime \prime}\right)$. There are five small filaments that look like small elongated granules. The three of them on the right side seem to make up one granule. In fact, this granule increases in brightness within the next five minutes and all of the five filaments that are visible at 08:32 UT disappear (see also, Hirzberger et al. 2002). But on the image at

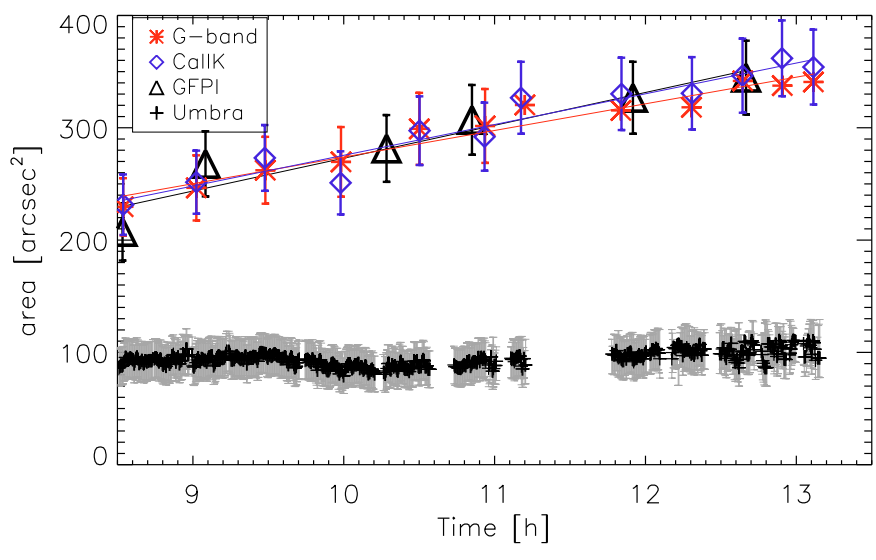

Fig. 4. Total and umbral areas of developing sunspot. Total area of spot is determined from manual contours for the various filtergrams. The straight lines result from corresponding least-squares fits. The black crosses correspond to the dark area of the umbra, with values around $100 \operatorname{arcsec}^{2}$.

09:33 UT, bright elongated points, resembling penumbral grains, become visible at the outskirts of the spot around 11:30 o'clock. These penumbral grains and the other filaments of case (ii) increase their radial and azimuthal extension gradually.

The penumbra grows from the four sections described in case (ii). In our G-band images, these growing filaments are characterized by having a brighter inner "grain" end and a darker tail farther outwards. The growth varies between the various sectors. At the section around 12:00 o'clock, there are no significant filaments at 08:32 UT. At 09:33 UT there are a few filaments with sizes from below $1^{\prime \prime}$ up to $2^{\prime \prime}$. At 10:32 UT the filaments in this section have a length from $3^{\prime \prime}$ to more than $4^{\prime \prime}$. At the end of the sequence, the penumbra has a radial extension of some $5^{\prime \prime}$. While the filaments increase in radial direction, the penumbra also develops in azimuthal direction. From the initial four sections we observe a gradual increase in azimuth until, at 13:06 UT, the penumbra encircles the umbra almost continuously from 7 o'clock clockwise to about 2 o'clock, corresponding to an azimuthal extend of $210^{\circ}$. The remaining $150^{\circ}$ of the circumference, where no stable penumbra forms, points towards the opposite polarity.

Growth suppressed towards opposite polarity: Elongated granules appear and disappear continuously in the area towards the opposite polarity. For example, at 09:33 UT there is a very thin bright filament at $\left(10^{\prime \prime},-9^{\prime \prime}\right)$, which resembles a penumbral filament, but it is well outside the spot. Like all the other filaments in this area, it is not stable and disappears. In fact, all elongated structures in this area have granular life times and are probably associated with flux emergence as observed in the numerical simulations of Cheung et al. (2008) and Tortosa-Andreu \& Moreno-Insertis (2009). This active emergence site seems to prevent a stable penumbra from forming.

Penumbra growth into surroundings: Comparing the images at 09:33 UT, 10:32, and 11:09, it is seen that the penumbral sections that are located away from the opposite polarity grow outwards. The inner bright filament heads stay on average at the same location, but the darker tails of the filaments increase radially in size outwards from the spot. As a result, the forming penumbra increases the total area of the spot. Figure 4 shows that the total area increases linearly with time by $130 \operatorname{arcsec}^{2}$ from 
$230 \operatorname{arcsec}^{2}$ up to $360 \operatorname{arcsec}^{2}$ within $275 \mathrm{~min}$. The umbra consists of (a) dark areas defined as having intensity values of less than $50 \%$ of the average quiet Sun and (b) light bridges. Since the area of the light bridges decreases slightly by $13 \mathrm{arcsec}^{2}$, while the dark area increases by about the same number (as seen in the lower curve with the black crosses in Fig. 4), the area of the umbra is almost constant during the time sequence. Therefore, the increase in the spot area is exclusively due to an increase in the penumbral area. The average radial extension of the penumbra amounts to about $6^{\prime \prime}$ at 13:06 UT.

Growth time scale: The area rate at which the penumbra forms can be estimated. For this we state that the area projection amounts to a factor of $\cos 26^{\circ}=0.9$ and that the net area of the umbra is about constant. Then the penumbral growth rate corresponds to $(130 / 0.9) \cdot(725 \mathrm{~km})^{2} /(275 \mathrm{~min}) \approx 4.6 \times 10^{3} \mathrm{~km}^{2} \mathrm{~s}^{-1}$. Individual penumbral sections form on a time scale of some $30 \mathrm{~min}$, but it takes an order of magnitude longer until a spatially extended penumbra develops. In our case it took a little less than $5 \mathrm{~h}$ to form a sunspot in which more than half of the umbra is surrounded by penumbral filaments.

Non-radial filaments: The snapshots display curved filaments, with different directions of curvature. Looking at the various subumbrae (areas separated by light bridges), the penumbral filaments do not point to a global center of the spot, but tend to point toward the centers of the local subumbra.

\section{Discussion and conclusions}

We acquired a 4:40 h time series of images with a temporal cadence good enough to resolve the dynamical time scale in the solar photosphere. Starting with a sunspot that has a few rudimentary penumbral filaments, we witnessed the formation of a penumbra and the simultaneous evolution of a light bridge.

The penumbra forms out of the leading spot of the active region. Individual filaments form on a time scale of $30 \mathrm{~min}$. For the penumbra to span more than half of the circumference it takes more than $4 \mathrm{~h}$. We find that the area increase of the sunspot exclusively stems from an increase of the penumbra. From this, following Martínez Pillet (1997), we conjecture that the initial spot area has reached a critical size. Any newly emerging magnetic flux that joins the spot increases the inclination of the magnetic field lines at the outer boundary. Then the inclination angle exceeds a critical value locally and an instability can form penumbral filaments. Assuming that the average magnetic field strength and inclination of the penumbra are about 1600 Gauss and $60^{\circ}$, respectively, we can use the rate of increasing area to estimate that the rate of increase for the magnetic flux of the spot corresponds to some $4 \times 10^{16} \mathrm{Mx} \mathrm{s}^{-1}$; i.e., at this emergence rate a sunspot with $10^{22} \mathrm{Mx}$ would be formed within 3 days. First results from our GFPI data indicate that the emerging magnetic flux of the leading polarity is merging with the leading sunspot (Schlichenmaier et al. 2010). Then, $4 \times 10^{16} \mathrm{Mx} \mathrm{s}^{-1}$ is also an estimate of the rate of the magnetic flux that emerges during the most active epoch of this active region.

The penumbra of our spot forms on the side opposite the following polarity of the active region. This is somewhat surprising since one may argue that, because of the loop structure of a bipolar active region, the inclination of the magnetic field on the side towards the opposite polarity is expected to be larger. And since a penumbra is a region of largely inclined field, it may preferentially form in such an environment. For the penumbra formation that we witnessed this argument does not hold.

On the side towards the opposite polarity, we observe (i) that individual filaments form, but are not stable and disappear on a dynamical time scale; and (ii) that elongated granules come and go, indicating a region of flux emergence. Since our spot increases in size, we speculate that the proper polarity of this emerging flux migrates towards and merges with the spot. This may also indicate why there is no stable penumbra on this side: Due to the ongoing flux emergence, this region is very active and dynamic such that the penumbra cannot settle down. In a forthcoming paper, we will evaluate our spectro-polarimetric data set, which was acquired simultaneously with the imaging data presented here, and study the properties of the flow and magnetic field in the region of flux emergence and the penumbra-forming region.

Acknowledgements. The German VTT is operated by the Kiepenheuer-Institut für Sonnenphysik at the Observatorio del Teide in Tenerife. We acknowledge the support by the VTT and KAOS group, Manolo Collados for setting up TIP, Christian Beck for advice and software, and Oliver Wiloth for assisting the observations. We are thankful to Wolfgang Schmidt and Michael Knölker for fruitful comments on the manuscript. NBG acknowleges the Pakt für Forschung, and RR the DFG grant Schm 1168/8-2.

\section{References}

Bello González, N., \& Kneer, F. 2008, A\&A, 480, 265

Bray, R. J., \& Loughhead, R. E. 1964, Sunspots (The International Astrophysics Series (London: Chapman \& Hall))

Cheung, M. C. M., Schüssler, M., Tarbell, T. D., et al. 2008, ApJ, 687, 1373

Collados, M., Lagg, A., Díaz García, J. J., et al. 2007, in The Physics of Chromospheric Plasmas, ed. P. Heinzel, I. Dorotovič, \& R. J. Rutten, ASP Conf. Ser., 368, 611

Hale, G. E., Ellerman, F., Nicholson, S. B., et al. 1919, ApJ, 49, 153

Hirzberger, J., Bonet, J. A., Sobotka, M., Vázquez, M., \& Hanslmeier, A. 2002, A\&A, 383, 275

Keppens, R., \& Martínez Pillet, V. 1996, A\&A, 316, 229

Leka, K. D., \& Skumanich, A. 1998, ApJ, 507, 454

Martínez Pillet, V. 1997, in 1st Advances in Solar Physics Euroconference. Advances in Physics of Sunspots, ed. B. Schmieder, J. C. del Toro Iniesta, \& M. Vazquez, ASP Conf. Ser. 118, 212

Martínez Pillet, V. 2002, Astron. Nachr., 323, 342

Puschmann, K. G., Kneer, F., Seelemann, T., et al. 2006, A\&A, 451, 1151

Rutten, R. J., Hammerschlag, R. H., Bettonvil, F. C. M., Sütterlin, P., \& de Wijn, A. G. 2004, A\&A, 413, 1183

Schlichenmaier, R., Bello González, N., Rezaei, R., et al. 2010, Astron. Nachr., submitted

Schlichenmaier, R., \& Collados, M. 2002, A\&A, 381, 668

Sobotka, M. 2003, Astron. Nachr., 324, 369

Solanki, S. K. 2003, A\&ARv, 11, 153

Strous, L. H., Scharmer, G., Tarbell, T. D., Title, A. M., \& Zwaan, C. 1996, A\&A, 306, 947

Tortosa-Andreu, A., \& Moreno-Insertis, F. 2009, A\&A, 507, 949

von der Lühe, O., Soltau, D., Berkefeld, T., et al. 2003, in SPIE Conf. Ser. 4853, ed. S. L. Keil, \& S. V. Avakyan, 187

Wöger, F., \& von der Lühe, O. 2008, in SPIE Conf. Ser., 7019

Yang, G., Xu, Y., Wang, H., et al. 2003, ApJ, 597, 1190

Zwaan, C. 1987, ARA\&A, 25, 83

Zwaan, C. 1992, in Sunspots. Theory and Observations, ed. J. H. Thomas \& N. O. Weiss, NATO ASIC Proc., 375, 75 